\title{
A closed-form solution to estimate space-dependent parameters in heat and mass transport
}

\author{
Ricky J. R. van Kampen ${ }^{1,2}$, Amritam Das ${ }^{3}$, Siep Weiland ${ }^{3}$ and Matthijs van Berkel ${ }^{1}$
}

\begin{abstract}
This paper presents a closed-form solution to estimate space-dependent transport parameters of a linear one dimensional diffusion-transport-reaction equation. The infinite dimensional problem is approximated by a finite dimensional model by 1) taking a frequency domain approach, 2) linear parameterization of the unknown parameters, and 3) using a semidiscretization. Assuming full state knowledge, the commonly used output error criterion is rewritten as the equation error criterion such that the problem results in linear least squares. The optimum is then given by a closed-form solution, avoiding computational expensive optimization methods. Functioning of the proposed method is illustrated by means of simulation.
\end{abstract}

\section{INTRODUCTION}

Heat and mass transport phenomena are widely studied in the domain of physics and chemistry. Examples include, but are not limited to, transport of thermal energy through nuclear fusion reactors [1], the study of the groundwatersurface interaction systems [2], and heat or moisture transport in buildings [3]. These phenomena are typically modeled around an operating point by linear parabolic partial differential equations (PDEs), commonly known as diffusiontransport-reaction equations [4]. In most physical diffusiontransport-reaction systems the -exact- parameters are often unknown. Hence, data-driven estimation of the unknown physical parameters is necessary to determine a model which can be used for simulation, analysis, prediction, and control.

Historically, the utilization of measured data to determine an estimate of -physical- parameters is known as an inverse problem [5], [6]; in contrast to forward problems where the model is used to generate data. Specifically, determining unknown physical parameters in dynamical systems is often considered as an optimization problem where an error criterion based on the mismatch between model and measurements is minimized. To this end, there are typically two sub-fields in PDE estimation: 1) grey-box identification where unknown parameters are estimated by minimizing the difference between a pre-selected model (class), e.g., a

\footnotetext{
${ }^{1}$ DIFFER - Dutch Institute for Fundamental Energy Research, Energy Systems and Control group, De Zaale 20, 5612 AJ Eindhoven, The Netherlands $\{$ R.J.R.vanKampen, M.vanBerkel $\}$ ediffer.nl

${ }^{2}$ Eindhoven University of Technology, Dept. of Mechanical Engineering, Control Systems Technology group, P.O. Box 513, 5600 MB Eindhoven, The Netherlands

${ }^{3}$ Eindhoven University of Technology, Dept. of Electrical Engineering, Control Systems group, P.O. Box 513, 5600 MB Eindhoven, The Netherlands $\{$ am.das, s.weiland $\}$ etue.nl

Acknowledgment: DIFFER is part of the institutes organisation of NWO. This work has been carried out within the framework of the EUROfusion Consortium and has received funding from the Euratom research and training programme 2014-2018 and 2019-2020 under grant agreement No 633053. The views and opinions expressed herein do not necessarily reflect those of the European Commission.
}

PDE with unknown diffusion, and measured data [7], [8]; 2) parameter learning which resorts to machine learning like techniques to avoid pre-selection of model-class [9], [10]. In both cases the underlying infinite dimensional models are generally approximated by finite dimensional models.

The standard method to estimate the unknown parameters is the output error criterion, i.e., taking the -weighted- sum of the squared error between the measurements and model output, primarily solved with iterative optimization methods [6]-[8], [11], [12]. These suffer from two problems: 1) they are solved iteratively, which is time consuming and in many cases the problem is non-convex, i.e., no guarantee for convergence to an optimal solution; 2) as only the output error is optimized and the unknown parameters are unconstrained, the parameters can start oscillating even when the output error is zero due to spatial aliasing of the state, which is estimated simultaneously. The latter is generally resolved by regularizing the unknown parameters [13]. However, this regularisation is often artificial, as there is usually no $a$ priori information on how the unknown parameters change as function of space. Hence, here we propose a different approach by separating the two problems. First, we estimate the solution of the state over space based on a finite number of measurements. This allows us to transform the output error in an equation error criterion. By transforming this criterion into the frequency domain, we can derive a closedform expression for the unknown parameters based on the estimated states, which is the novelty of this paper. Hence, we can uniquely and directly -without iteration- determine the diffusivity, convectivity, reactivity, and the source -as function of space-, simultaneously. Combining this with advances in 1) modern frequency domain signal processing to reduce noise and removing the initial condition [14], [15]; 2) recent innovations in dealing with experimentally unknown boundary conditions [16]; 3) a wide variety of functions to spatially parameterize transport [17]. This results in a highly versatile and fast method to acquire reliable estimates of the spatially varying parameters. This is in strong contrast to direct solutions proposed in the literature which are based on (piecewise) constant parameters [18] and often only consider diffusion [19], [20].

\section{PROBLEM FORMULATION}

The estimation of space-dependent physical parameters in heat and mass transport is performed based on the following specifications.

a) Model-class: A class of linear parabolic PDEs is considered in a one dimensional bounded spatial geometry 
to model the spatio-temporal dynamics of heat and mass transport. For all $x \in \mathbb{X}:=\left[x_{b}, x_{e}\right] \subset \mathbb{R}$ and $t \in \mathbb{T}:=$ $\left[t_{0}, \infty\right) \subseteq \mathbb{R}_{>0}$, the class of PDEs is defined as

$$
\frac{\partial z}{\partial t}=D(x) \frac{\partial^{2} z}{\partial x^{2}}+V(x) \frac{\partial z}{\partial x}+K(x) z+P(x) s(t) .
$$

Here, the state $z: \mathbb{X} \times \mathbb{T} \rightarrow \mathbb{R}$ is a multi-variable function (that can describe temperature or mass concentration) and (1) is understood point-wise in $x \in \mathbb{X}$ and $t \in \mathbb{T}$ with $z$ evaluated as $z(x, t)$. The physical transport parameters are diffusivity $D: \mathbb{X} \rightarrow \mathbb{R}_{>0}$, convectivity $V: \mathbb{X} \rightarrow \mathbb{R}$, and reactivity $K: \mathbb{X} \rightarrow \mathbb{R}$. The external input is denoted by $s: \mathbb{T} \rightarrow \mathbb{R}$. Furthermore, the spatial distribution of the input is given by the function $P: \mathbb{X} \rightarrow \mathbb{R}$.

For well-posedness, the PDE is constrained by two boundary conditions at locations $x_{b}$ and $x_{e}$. Moreover, the initial condition $z\left(\cdot, t_{0}\right)$ is assumed compatible with the model and its boundaries.

b) Measured Data: Corresponding to the heat and mass transport phenomena, location specific values of the state function $z$ are measured over time and available as data. Let there be $M>0$ sensors that measure $z$ at the locations given by the set of points $\mathbb{X}_{M}:=\left\{\check{x}_{1}, \check{x}_{2}, \ldots, \check{x}_{M}\right\} \subset \mathbb{X}$. The measured output signals are $y^{m}(t)=z\left(\check{x}_{m}, t\right)$ for all $t \in \mathbb{T}$, with $m \in\{1, \ldots, M\}$. The input $s(t)$ is assumed to be known or measured for all $t \in \mathbb{T}$.

c) Problem Formulation: In practical applications, the explicit definitions of space-dependent transport parameters $\{D(x), V(x), K(x), P(x)\}$ are often not available. Moreover, the boundary conditions that constrain heat and mass transport phenomena may depend on the spatially varying transport parameters, and, hence, remain unknown. In a similar fashion, in practice, the -exact- initial condition $z\left(\cdot, t_{0}\right)$ may also be unknown. Therefore, this paper takes the extremum measurements as boundary inputs, however, other linear boundary conditions are also allowed.

To complete the model in (1), the spatially varying transport parameters $\{D(x), V(x), K(x), P(x)\}$ have to be estimated based on measured data. This results in the following estimation problem:

Problem 1. Given the pre-processed data-set (see Remark 2)

$$
\mathbb{D}:=\left\{y^{1}(t), \ldots, y^{M}(t), s(t) \mid t \in\left[t_{0}, \infty\right)\right\},
$$

estimate the unknown function $\gamma: \mathbb{X}_{E} \rightarrow \mathbb{R}^{4}$,

$$
\gamma(x):=\operatorname{col}(D(x), V(x), K(x), P(x)),
$$

by minimizing a cost function $\mathcal{V}(\mathbb{D}, z(x, t ; \gamma))$-defined in Sec IV-over $\gamma$ such that the solution $z(x, t ; \gamma)$ satisfies the model (1) with parameters $\gamma$ in the sense that

$$
\frac{\partial z}{\partial t}=\operatorname{row}\left(\frac{\partial^{2} z}{\partial x^{2}}, \frac{\partial z}{\partial x}, z, s\right) \gamma,
$$

subject to the boundary conditions and initial condition

$$
z\left(\check{x}_{1}, t\right)=y^{1}(t), \quad z\left(\check{x}_{M}, t\right)=y^{M}(t), \quad z\left(x, t_{0}\right)=0 .
$$

Remark 1. Using the extremum measurements as Dirichlet boundary conditions reduces the estimation domain to
$\mathbb{X}_{E}:=\left[\check{x}_{1}, \check{x}_{M}\right]$ [16]. Moreover, this allows to set-up the parameter estimation problem without the need of full knowledge about the actual boundary conditions.

Remark 2. With -advanced- signal processing techniques, the measured signal can be split into a transients/drift signal (non-steady-state behavior, e.g. from the initial condition), a periodic signal (from the excitation) and additive (filtered) noise [14], [15]. By removing the transient and noise terms from the original signal, only the periodic signal remains in the filtered data set $\mathbb{D}$, which is equivalent to $z\left(\cdot, t_{0}\right)=0$.

\section{FINITE DIMENSIONAL FREQUENCY DOMAIN PROBLEM}

Problem 1 is infinite dimensional with no known analytic solution for $z(x, t)$. Therefore, this section approximates the infinite dimensional problem by creating a finite dimensional model following the methodology from [17].

\section{A. Frequency domain approach}

Assuming that the discrete Fourier transformed input $S(k)$ has (excited) frequency bins $k \in \mathbb{K}$, Problem 1 can be studied in the frequency domain without loss of information (Parseval's Theorem). Moreover, due to linearity of the model, $Z(x, k)$ is independent for each excited bin $k$. The frequency domain model of (3) is then given by

$$
\begin{aligned}
i \omega_{k} Z & =\operatorname{row}\left(\frac{\partial^{2} Z}{\partial x^{2}}, \frac{\partial Z}{\partial x}, Z, S\right) \gamma \\
Y(k) & :=\operatorname{col}\left(Z\left(\check{x}_{2}, k\right), \ldots, Z\left(\check{x}_{M-1}, k\right)\right)
\end{aligned}
$$

subject to the boundary conditions

$$
Z\left(\check{x}_{1}, k\right)=Y^{1}(k), \quad Z\left(\check{x}_{M}, k\right)=Y^{M}(k),
$$

with discrete Fourier transformed state $Z: \mathbb{X} \times \mathbb{K} \rightarrow \mathbb{C}$, input $S: \mathbb{K} \rightarrow \mathbb{C}$, output $Y(k), i^{2}=-1$ and angular frequency $\omega_{k}$ corresponding to the $k^{\text {th }}$-bin.

Remark 3. In excitation experiments only a finite number of bins are informative, i.e., those bins which are present in the input $S(k)$ and are above the noise level [14]. Hence, in practice only a-few-finite number of bins need to be considered (see [21] for details).

\section{B. Linear parameterization of the unknown functions}

For estimation purposes, assume that $\gamma$ belongs to a function space $\Gamma$ that is parameterized by a surjective mapping $\Pi: \Theta \rightarrow \Gamma$ that is described by a finite sum of basis functions $B_{r}(x):=\operatorname{diag}\left(B_{r}^{D}(x), B_{r}^{V}(x), B_{r}^{K}(x), B_{r}^{P}(x)\right)$,

$$
\gamma(x ; \theta):=[\Pi(\theta)](x):=\sum_{r=1}^{R} B_{r}(x) \theta_{r},
$$

with $\theta=\operatorname{col}\left(\theta_{1}, \ldots, \theta_{R}\right)$ and $\theta_{r}=\operatorname{col}\left(\theta_{r}^{D}, \theta_{r}^{V}, \theta_{r}^{K}, \theta_{r}^{P}\right) \in$ $\Theta \subset \mathbb{R}^{4}$. With this parameterization, the estimation of $\gamma$ amounts to estimating $\theta \in \mathbb{R}^{4 R}$. 


\section{Semi-discretization}

The infinite dimensional model (4) is approximated by a central finite difference scheme that converges to the exact solution for $N \rightarrow \infty$ [22]. The finite dimensional model is

$$
\begin{aligned}
i \omega_{k} \mathbf{Z}(k) & =A(\theta) \mathbf{Z}(k)+B(\theta) U(k) \\
Y(k) & =C \mathbf{Z}(k)
\end{aligned}
$$

with state vector $\mathbf{Z}(k):=\operatorname{col}\left(Z\left(x_{2}, k\right), \ldots, Z\left(x_{N-1}, k\right)\right)$ at sample $x_{j} \in \mathbb{X}_{d} \subset \mathbb{X}, j \in\{1, \ldots, N\}$, extended input vector $U(k)=\operatorname{col}\left(S(k), Y^{1}(k), Y^{M}(k)\right)$. Here, $A(\theta)$ and $B(\theta)$ contain the boundary conditions, are linear affine in $\theta$, and defined in [17]. The -observation- matrix $C$ maps states to output. Altogether, the finite dimensional problem yields

Problem 2. Given the pre-processed data-set

$$
\mathbb{D}=\left\{\operatorname{col}\left(Y^{1}(k), \ldots, Y^{M}(k)\right), S(k) \mid k \in \mathbb{K}\right\} .
$$

Estimate $\theta$ by minimizing a cost function $\mathcal{V}(\mathbb{D}, \mathbf{Z}(k ; \theta))$ over $\theta$ such that $\mathbf{Z}(k ; \theta)$ satisfies the model (6).

\section{THE INVERSE PROBLEM}

This section describes our methodology to estimate the unknown weights. In the literature for distributed parameter systems, different error criteria are proposed to calculate the parameters. The commonly used criterion is the output error criterion [6]-[8], [11], [17] and the rarely used equation error criterion [7] which we will use in the frequency domain to derive a closed-form solution for the unknown parameters.

\section{A. Output error criterion}

The commonly used cost function is the output error criterion which is the sum of the squared error between solution $z(x, t ; \gamma)$ for a given $\gamma$ and measurements $y(t)$, i.e.,

$$
\mathcal{V}_{\mathrm{oe}}(\theta):=\int_{t_{0}}^{\tau} \int_{x_{b}}^{x_{e}}|y(t)-\mathfrak{C} z(x, t ; \gamma(x ; \theta))|^{2} \mathrm{~d} x \mathrm{~d} t
$$

with observation map $\mathfrak{C}$ that maps state to output. This criterion is transformed into the frequency domain such that the output error criterion for Problem 2 and [17] is given by

$$
\mathcal{V}_{\mathrm{oe}}(\theta)=\sum_{k \in \mathbb{K}}\left\|Y_{k}-C\left(i \omega_{k} I-A(\theta)\right)^{-1} B(\theta) U_{k}\right\|^{2}
$$

with shorthand notation $Y_{k}=Y(k)$ and $U_{k}=U(k)$. This criterion is nonlinear in $\theta$ due to the inverse and multiplication. As such needs to be iteratively optimized with often no guarantee for convergence to the global minimum. As this is generally the case for the output error criterion, we propose to use the equation error criterion for which a closed-form solution can be derived.

\section{B. Equation error criterion}

In the equation error criterion, the state of the model is replaced by measurements or estimates of the state [7], such that for (3), the equation error criterion is defined as

$$
\begin{aligned}
& \mathcal{V}_{\mathrm{ee}}(\theta):= \\
& \int_{t_{0}}^{\tau} \int_{\check{x}_{1}}^{\check{x}_{M}}\left(\frac{\partial y}{\partial t}-\operatorname{row}\left(\frac{\partial^{2} y}{\partial x^{2}}, \frac{\partial y}{\partial x}, y, s\right) \gamma(x ; \theta)\right)^{2} \mathrm{~d} x \mathrm{~d} t
\end{aligned}
$$

This can be simplified in the frequency domain: the integral over time simplifies to a summation over the excited frequency bins $k \in \mathbb{K}$ without loss of information (Parseval's theorem). Assuming that the states are measured or estimated by $\hat{Z}_{k}$, the equation error for Problem 2 simplifies to

$$
\mathcal{V}_{e e}(\theta)=\sum_{k \in \mathbb{K}}\left\|i \omega_{k} \hat{Z}_{k}-\left(A(\theta) \hat{Z}_{k}+B(\theta) U_{k}\right)\right\|^{2} .
$$

\section{Derivation of the closed-form solution}

Consider the equation error criterion in (10) and that $A(\theta)$ and $B(\theta)$ are linear affine in $\theta$, then new matrices $\tilde{A}, \tilde{B}$ can be defined as a function of the data $U_{k}$ and $\hat{Z}_{k}$ such that $A(\theta) \hat{Z}_{k}=\hat{A}\left(\hat{Z}_{k}\right) \theta$ and $B(\theta) U_{k}=\hat{B}\left(U_{k}\right) \theta$ (see Appendix). As a result, (10) can be written as

$$
\mathcal{V}_{\mathrm{ee}}=\sum_{k \in \mathbb{K}}\left\|i \omega_{k} \hat{Z}_{k}-\left(\hat{A}\left(\hat{Z}_{k}\right)+\hat{B}\left(U_{k}\right)\right) \theta\right\|^{2}
$$

with the closed-form solution

$$
\hat{\theta}:=\left((\bar{A}+\bar{B})^{\mathrm{H}}(\bar{A}+\bar{B})\right)^{-1}(\bar{A}+\bar{B})^{\mathrm{H}} \bar{Y},
$$

where ${ }^{\mathrm{H}}$ denotes the Hermitian transpose, $\bar{A}, \bar{B}$, and $\bar{Y}$ are the column concatenation of $\hat{A}\left(\hat{Z}_{k}\right), \hat{B}\left(U_{k}\right)$, and $i \omega_{k} \hat{Z}_{k}$ for all excited bins $k \in \mathbb{K}$, respectively. Hence, the optimal weighting for $\theta$, and thus $\gamma(x)$, are determined without using iterative optimization methods.

Remark 4. The equation error in combination with different cost functions such as weighted and total linear least squares also have closed-form solutions [23].

\section{State estimation}

A unique solution requires knowledge of the state at each discretization point, i.e. a "space-"continuous measurement. If $N \rightarrow \infty$, the finite dimensional description converges to the true infinite dimensional solution and an exact solution can be found.

However, due to limited spatial measurements, the purpose is to estimate the full state based on these measurements. This raises the fundamental problem of unknown in-between sensor behavior. In case of regularized output error [7], the intermediate relationship in-between measurements is determined by the regularized functions for the transport parameters. This technique is reminiscent to the (spatial) Nyquist-Shannon (NS) sampling theorem. Therefore, a consistent -spatial- signal reconstruction using the NS theorem is required and currently being worked out. Alternatively, the states can be inferred by interpolating the measurements using machine learning techniques -currently being further worked out- [24], [25]; classic interpolation methods; or reducing the number of states in the model to match the measurements. Note that the selected method should be model free such that the problem remains affine in the unknown parameters. In the simulation section, we show the latter two approximation methods in combination with the closed-form solution. 


\section{SIMULATION RESULTS}

In this section, two simulation scenarios are presented demonstrating the merit of the proposed methodology. As the here proposed method has a closed-form solution that only requires solving a linear matrix equality, it is expected to be significantly faster than iterative optimization methods, e.g., when compared to [17]. This can be exploited by considering two scenarios: scenario 1 which estimates $\{D(x), P(x)\}$ testing different orders (weights) $R$ of the basis functions and scenario 2 which tests different interpolation density of the data when all four functions $\{D(x), V(x), K(x), P(x)\}$ are estimated using fixed set of basis functions $B_{r}(x)$ and their orders $R$.

\section{A. Data generation}

The simulation example is inspired by perturbative experiments in the field of nuclear fusion [26]. The heat transport, (1), is generally analyzed on the normalized domain $\mathbb{X}=\left[x_{b}, x_{e}\right]=[0,1]$ of the -minor- plasma radius. Here $x_{b}$ is at the center and $x_{e}$ is at the edge of the plasma. The corresponding boundary conditions in the simulation are $\frac{\partial Z}{\partial x}\left(x_{b}, \cdot\right)=0$ due to (axi)symmetry and Dirichlet boundary condition $Z\left(x_{e}, \cdot\right)=0$ due to a significant temperature difference between core plasma $\sim 170$ million ${ }^{\circ} \mathrm{C}$ and edge plasma $\sim 1$ million ${ }^{\circ} \mathrm{C}$. Typical functions used in nuclear fusion are $D^{\operatorname{sim}}(x)=5 x^{3}-0.005 x+5$, $V^{\operatorname{sim}}(x)=15 x^{2}-0.005, K^{\operatorname{sim}}(x)=-3 x$, and $P^{\operatorname{sim}}(x)=$ $0.2+\frac{7}{\sqrt{\pi}} \exp \left(\frac{-(x-0.35)^{2}}{(0.1)^{2}}\right)+\frac{5.6}{\sqrt{\pi}} \exp \left(\frac{-(x-0.6)^{2}}{(0.1)^{2}}\right)$. For the perturbation of the plasma temperature a microwave source is used, where the excitation signal $S(\omega)$ is a block-wave of $\omega_{0}=50 \pi$ with a $70 \%$ duty cycle. Here, only the first five harmonics $\omega_{k}=k \omega_{0}, k=1, \ldots, 5$ have a significant contribution and are used for the estimation. The temperature data is generated by a simulation with a central finite difference grid of $N=801$ sample points.

\section{B. Estimation of $\{D(x), P(x)\}$ with unknown order $R$}

In scenario 1 , the goal is to estimate $\{D(x), P(x)\}$ without knowledge on the correct orders $\left(R^{D}, R^{P}\right)$ in (5), which practically means we do not know the shape of $\{D(x), P(x)\}$. For ease of explanation, we set $V(x)=$ $K(x)=0$ for this example. As the number of sensors plays an important role, we choose here $M=22$ sensors that are located at $\check{x}_{m}=0.05+0.04125(m-1)$, with $m=1, \ldots, M$. This corresponds to the electron cyclotron emission (ECE) diagnostic that measures in a medium sized fusion reactor.

In principle, any basis function can be used and several can be tested simultaneously. Here, we use -arbitrarily chosenChebyshev polynomials for both parameters [22].

We vary the orders $R^{D}, R^{P} \in\{1, \ldots, 30\}$ and use a discretization grid that equals the measurement grid $\mathbb{X}_{d}=$ $\mathbb{X}_{M}$, thus $C=I$. Naturally, the cost (10) decreases for increasing $R^{D}$ and $R^{P}$ as shown in Fig. 1. For three different combinations, i.e., $\left(R^{D}, R^{P}\right)=(6,9),(9,16)$, and $(22,24)$ the resulting estimates are shown in Fig. 2.

Fig. 2 shows that when the order $\left(R^{D}, R^{P}\right)$ is too low, e.g., for $(6,9)$, significant errors occur in the estimates. If

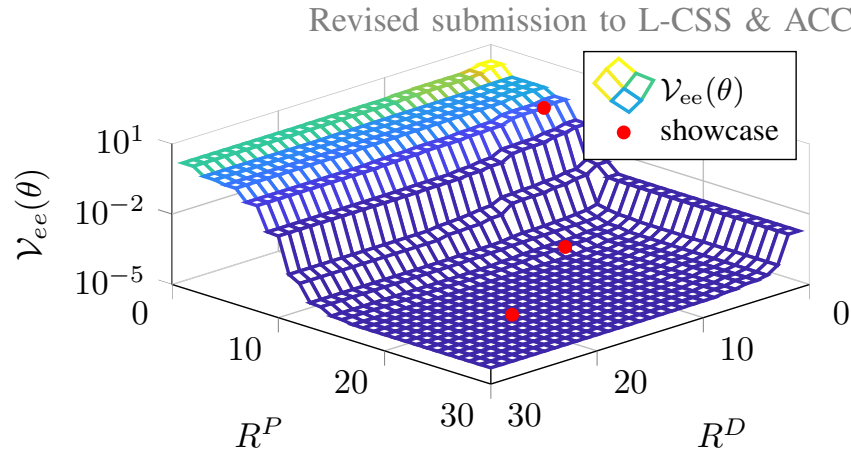

Fig. 1: The fitting error map $\mathcal{V}_{\mathrm{ee}}(\theta)$ for scenario 1 by approximating the parameters $\{D(x), P(x)\}$ with different number of basis-functions, $R^{D}, R^{P}$, respectively. Here, the - annotates the locations $\left(R^{D}, R^{P}\right)=(4,5),(9,16)$, and $(22,24)$ shown in Fig. 2.
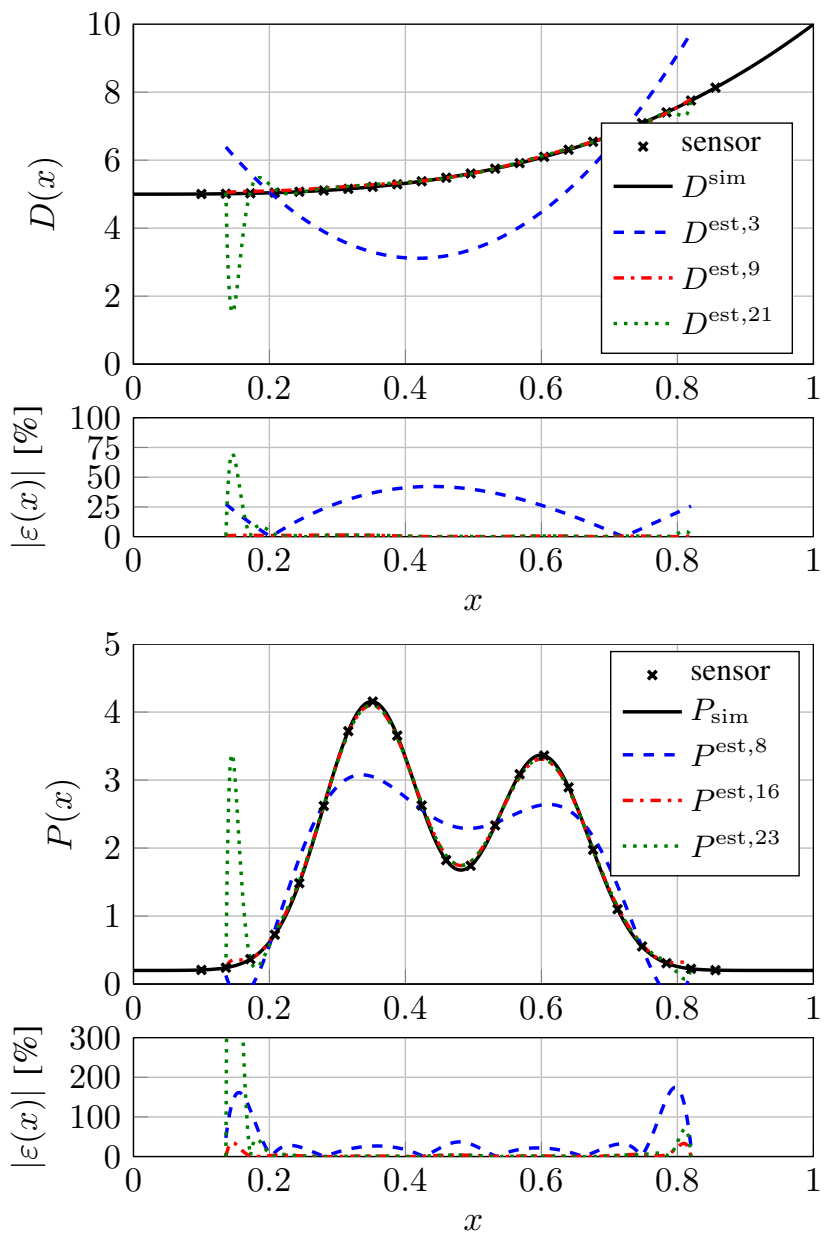

Fig. 2: The estimated parameters $\{D(x), P(x)\}$ over $\mathbb{X}_{E}$ for scenario 1 with the number of basis-function $\left(R^{D}, R^{P}\right)=$ $(6,9),(9,16)$ and $(22,24)$ and their relative error $\varepsilon(x)$.

the order is sufficiently high, e.g., for the pairs $(9,16)$ and $(22,24)$, the estimates have the correct value at the sensor locations as is imposed by the equation error. Consequently, only the values of $\gamma(x)$ at the sensor locations should be considered as a correct result given that the combination of basis functions and order give sufficient freedom. 
Remark 5. Although the intermediate points between sensor locations should not be considered in this methodology, $\gamma(x)$ is defined at these points and plotted for completeness. As the comparison of the orders $(9,16)$ and $(22,24)$ shows, the finite dimensional approximation does not pose a unique solution for $\gamma(x)$ at the intermediate points. This can be resolved by increasing the discretization grid, e.g. by interpolating the data, which is further investigated in Sec. V-C.

\section{Estimation using spatial interpolations of the temperature}

In the simulation for scenario 2, we investigate the effect of interpolations on the estimation. In scenario 2 all the functions are non-zero and according to Sec. V-A. Here, we use a sensor grid which corresponds to that of a larger fusion reactor, i.e., the temperature is measured by $M=60$ sensors located at $\check{x}_{m}=0.1625 m$, with $m=1, \ldots, M$.

In this scenario, the order of the basis functions is fixed and only the weights are estimated. Polynomial basis functions are used for $\{D(x), V(x), K(x)\}$ and Bspline functions for $P(x)$ to approximate Gaussians. The choice of order for the monomial basis functions to estimate $\{D(x), U(x), K(x)\}$ is higher than the actual order and is chosen to be $R^{D}=8, R^{V}=6$ and $R^{K}=3$ such that it is possible to find an exact description. The basis function for $P(x), B_{r}^{B}(x)$ is designed using the De Boor's algorithm [27] with 58 equally distributed control points, thus $R^{P}=58$.

Three estimations are presented where, 1) the discretization grid equals the measurement grid $N_{1}=M, 2$ ) the measurement data is interpolated over space to generate artificial spatial measurements, i.e., $N_{2}=2(M-1)+1$, and 3) more interpolation points, i.e. $N_{3}=10(M-1)+1$. The data is interpolated using cubic splines.

The estimation results are shown in Fig. 3. Overall, the estimated parameters $\{D(x), P(x)\}$ closely match with the simulated parameters, while $\{V(x), K(x)\}$ are estimated with a significantly lower accuracy. Estimating $\{V(x), K(x)\}$ requires a higher accuracy of the discretization grid. Therefore, interpolating the data increases the accuracy (see results for $N_{2}$ ), however, over-interpolation can affect the estimation accuracy negatively (see results for $N_{3}$ ).

\section{CONCLUSIONS \& DISCUSSION}

This paper presents a novel method to efficiently estimate the unknown space-dependent transport parameters, based on a closed-form solution of the equation error criterion. The closed-form solution is formulated as a linear matrix equality such that high density grids can be solved computationally efficient. As a result, if the states $z(\cdot, t)$ are known, the parameters are estimated uniquely with the desired accuracy by taking a sufficiently dense discretization grid.

In practice, measurements are often only provided at a limited set of spatial locations. This is generally resolved by (i) applying regularization or restrictions on the unknown parameters or (ii) as we have done here by "interpolating" the measurements to increase the grid density. There are three reasons why we prefer approach (ii): 1) As we are estimating the unknown parameters, prior information on the -smoothness of- parameters is generally unavailable, whereas smoothness on the states is required due to the underlying parabolic PDE; 2) the states as function of space can easily be -visually- inspected for correctness and validated by taking additional spatial measurements; and 3) a closedform solution can be used which significantly speeds up the process and avoids convergence to a local minimum.

\section{APPENDIX}

The matrices used in (11) are given by

$$
\begin{aligned}
\hat{A}(\mathbf{Z}(k)):= & {\left[L_{1}^{D}, L_{1}^{V}, L_{1}^{K}, \mathbf{0}, \ldots,\right.} \\
& \left.L_{R}^{D}, L_{R}^{V}, L_{R}^{K}, \mathbf{0}\right]\left(I_{4 R} \otimes \mathbf{Z}(k)\right), \\
\hat{B}(U(k)):= & {\left[\mathbf{0}, g_{1}^{D}, h_{1}^{D}, \mathbf{0}, g_{1}^{V}, h_{1}^{V}, \mathbf{0}, \mathbf{0}, \mathbf{0}, f_{1}^{P}, \mathbf{0}, \mathbf{0}, \ldots,\right.} \\
& \left.\mathbf{0}, g_{R}^{D}, h_{R}^{D}, \mathbf{0}, g_{R}^{V}, h_{R}^{V}, \mathbf{0}, \mathbf{0}, \mathbf{0}, f_{R}^{P}, \mathbf{0}, \mathbf{0}\right] \\
& \left(I_{4 R} \otimes U(k)\right),
\end{aligned}
$$

where $\otimes$ denotes the Kronecker product and $\mathbf{0}$ the zero vector/matrix of the appropriate size. The central finite difference matrices $L$ with grid sample $\Delta_{x}>0$ are

$$
L_{r}^{D}:=\frac{1}{\left(\Delta_{x}\right)^{2}} \tilde{B}_{r}^{D}\left[\begin{array}{cccc}
-2 & 1 & & \\
1 & -2 & 1 & \\
& \ddots & \ddots & \ddots \\
& & 1 & -2
\end{array}\right],
$$

Here, $\tilde{B}_{r}^{D}, \tilde{B}_{r}^{V}$ and $\tilde{B}_{r}^{K}$ are diagonal matrices of dimension $(N-2) \times(N-2)$ with the diagonal entries $B_{r}^{D}\left(x_{j}\right)$, $B_{r}^{V}\left(x_{j}\right), \quad B_{r}^{K}\left(x_{j}\right)$ evaluated at each grid point $x_{j}$, $j \in\{2, \ldots, N-1\}$. The vectors for the input and boundary conditions are $g_{r}^{D}:=\operatorname{col}\left(\frac{B_{r}^{D}\left(x_{2}\right)}{\left(\Delta_{x}\right)^{2}}, 0, \ldots, 0\right), g_{r}^{U}:=$ $\operatorname{col}\left(-\frac{B_{r}^{V}\left(x_{2}\right)}{2 \Delta_{x}}, 0, \ldots, 0\right), h_{r}^{D}:=\operatorname{col}\left(0, \ldots, 0, \frac{B_{r}^{D}\left(x_{N-1}\right)}{\left(\Delta_{x}\right)^{2}}\right)$, $h_{r}^{U} \quad:=\operatorname{col}\left(0, \ldots, 0, \frac{B_{r}^{V}\left(x_{N-1}\right)}{2 \Delta_{x}}\right), \quad$ and $f_{r}^{P} \quad:=$ $\operatorname{col}\left(B_{r}^{P}\left(x_{2}\right), \ldots, B_{r}^{P}\left(x_{N-1}\right)\right)$.

\section{REFERENCES}

[1] M. van Berkel, G. W. Oosterwegel, M. Anthonissen, H. J. Zwart, and G. Vandersteen, "A novel frequency domain maximum likelihood approach for estimating transport coefficients in cylindrical geometry for nuclear fusion devices," in Proc. IEEE Conf. Decis. Control, pp. 3220-3226, 2019.

[2] U. Schneidewind et al., "LPMLE3: A novel 1-D approach to study water flow in streambeds using heat as a tracer," Water Resour. Res., vol. 52, no. 8, pp. 6596-6610, 2016.

[3] M. Irsyad, A. D. Pasek, Y. S. Indartono, and A. W. Pratomo, "Heat transfer characteristics of building walls using phase change material," IOP Conf. Ser.: Earth Environ. Sci., vol. 60, p. 012028, Mar. 2017.

[4] F. P. Incropera, D. P. Dewitt, T. L. Bergman, and A. S. Lavine, Fundamentals of Heat and Mass Transfer. Hoboken, USA: Wiley, 6th ed., 2006.

[5] A. Tarantola, Inverse Problem Theory and Methods for Model Parameter Estimation. Philadelphia, USA: SIAM, 2004.

[6] A. Kirsch, An Introduction to the Mathematical Theory of Inverse Problems. New York, USA: Springer, 2nd ed., 2011.

[7] H. Banks and K. Kunisch, Estimation Techniques for Distributed Parameter Systems. Boston, USA: Birkhäuser, 1989. 
Revised submission to L-CSS \& ACC
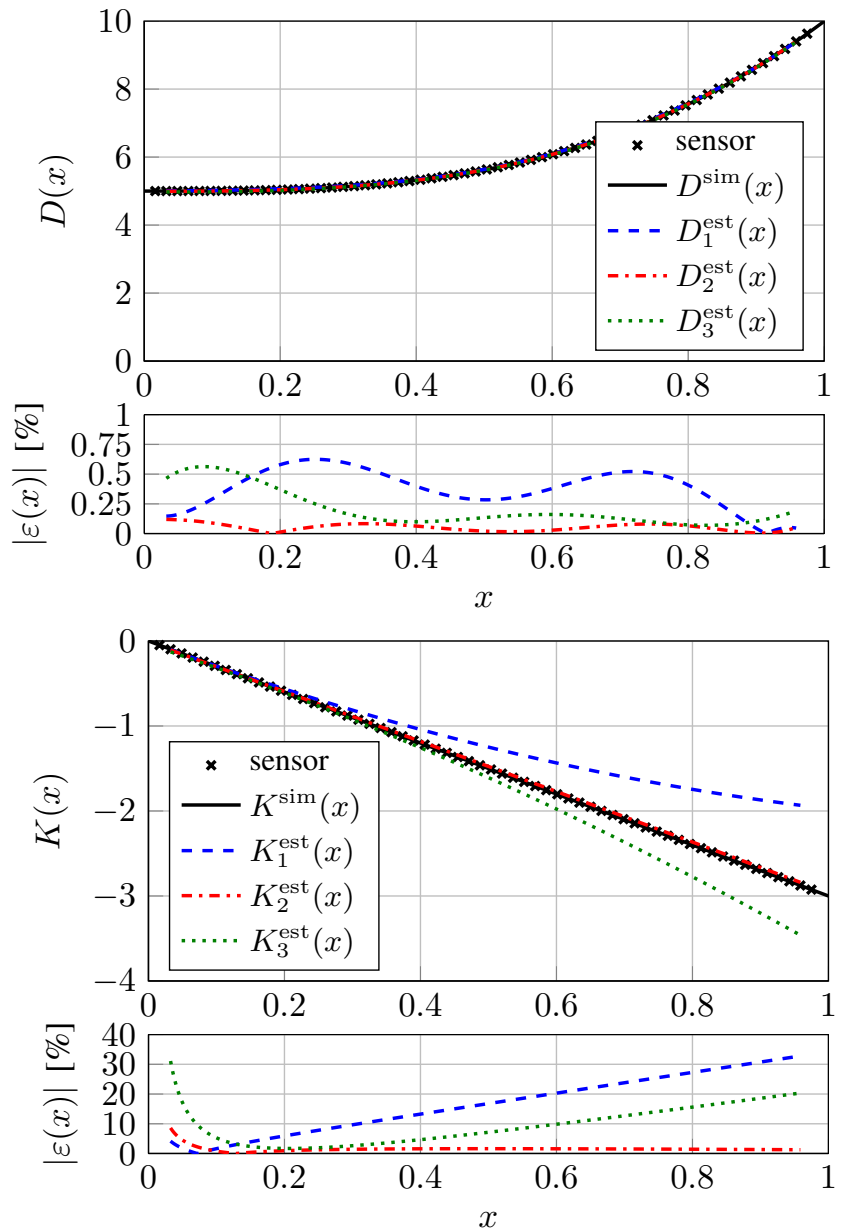
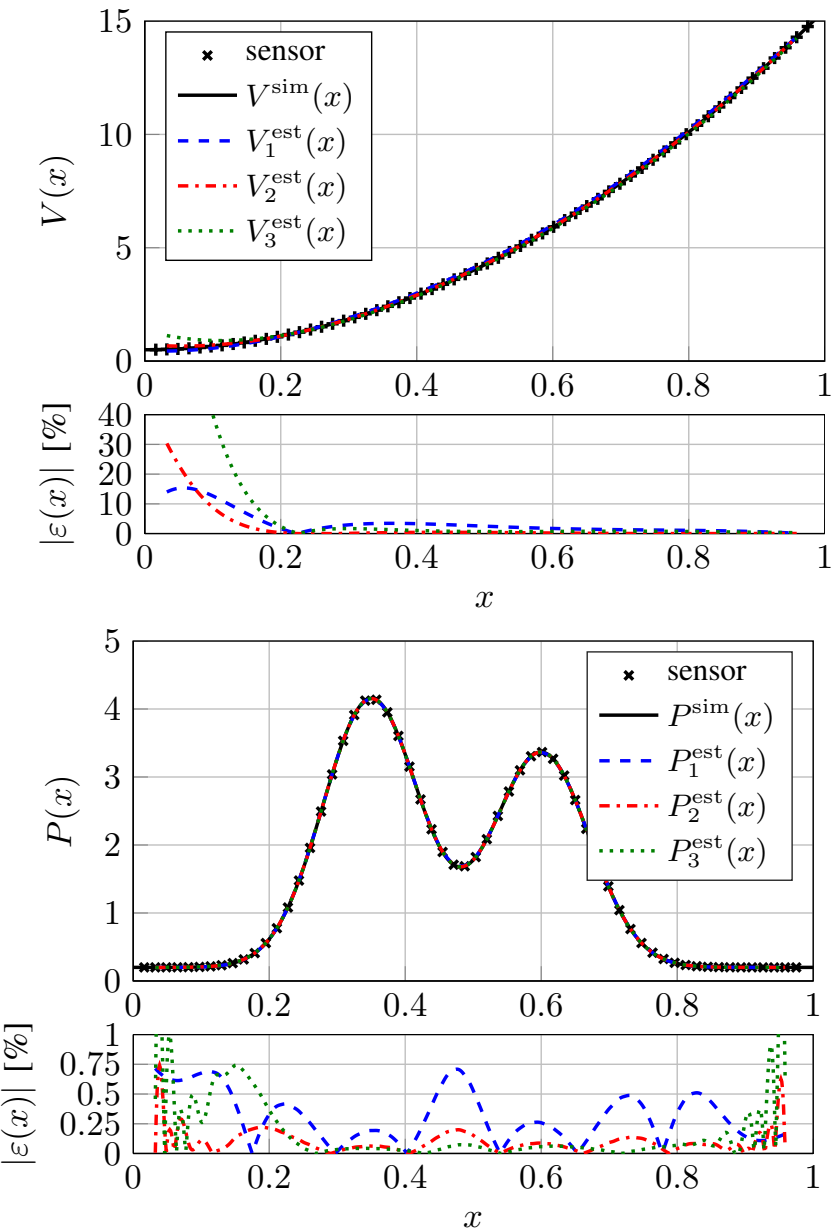

Fig. 3: Estimated parameters $\{D(x), V(x), K(x), P(x)\}$ over $\mathbb{X}_{E}$ for scenario 2 and the relative error $\varepsilon(x)$ between the simulated parameter and the estimated parameter. The subscript 1,2,3 denote if the estimates are based on the measurements $N_{1}=M$, or the interpolated measurements $N_{2}=2(M-1)+1$ or $N_{3}=10(M-1)+1$, respectively.

[8] C. R. Vogel, Computational Methods for Inverse Problems. Philadelphia, USA: SIAM, 2002.

[9] X. Xun, J. Cao, B. Mallick, A. Maity, and R. J. Carroll, "Parameter estimation of partial differential equation models," J. Am. Stat. Assoc., vol. 108, no. 503, pp. 1009-1020, 2013.

[10] A. M. Tartakovsky, C. Ortiz Marrero, P. Perdikaris, G. D. Tartakovsky, and D. Barajas-Solano, "Learning Parameters and Constitutive Relationships with Physics Informed Deep Neural Networks," arXiv $e$ prints, p. arXiv:1808.03398, 2018.

[11] H. G. Bock, T. Carraro, W. Jäger, S. Körkel, R. Rannacher, and J. P. Schlöder, Model Based Parameter Estimation. Berlin; Heidelberg, Germany: Springer, 2013.

[12] S. Mechhoud, E. Witrant, L. Dugard, and D. Moreau, "Estimation of heat source term and thermal diffusion in tokamak plasmas using a Kalman filtering method in the early lumping approach," IEEE Trans. Control Syst. Technol., vol. 23, no. 2, pp. 449-463, 2015.

[13] K. Ito and K. Kunisch, Lagrange Multiplier Approach to Variational Problems and Applications. Philadelphia, USA: SIAM, 2008.

[14] R. Pintelon and J. Schoukens, System Identification: A Frequency Domain Approach. Hoboken, USA: Wiley, 2nd ed., 2012.

[15] M. van Berkel et al., "Correcting for non-periodic behaviour in perturbative experiments: application to heat pulse propagation and modulated gas-puff experiments," Plasma Phys. Control. Fusion, vol. 62, no. 9, p. 094001, 2020

[16] M. van Berkel, G. Vandersteen, E. Geerardyn, R. Pintelon, H. Zwart, and M. de Baar, "Frequency domain sample maximum likelihood estimation for spatially dependent parameter estimation in PDEs," Automatica, vol. 50, no. 8, pp. 2113-2119, 2014.

[17] A. Das, S. Weiland, and M. van Berkel, "Frequency Domain Estimation of Spatially Varying Parameters in Heat and Mass Transport," 2019 Am. Control Conf., pp. 600-605, 2019.
[18] M. van Berkel et al., "Explicit approximations to estimate the perturbative diffusivity in the presence of convectivity and damping. I. Semi-infinite slab approximations," Phys. Plasmas, vol. 21, no. 11, p. 112507, 2014.

[19] R. E. Ewing and T. Lin, "A direct method for parameter estimation in a hyperbolic partial differential equation," in Proc. 27th Conf. Decis. Control, pp. 1662-1667, 1988.

[20] W. Liao, M. Dehghan, and A. Mohebbi, "Direct numerical method for an inverse problem of a parabolic partial differential equation," $J$. Comput. Appl. Math., vol. 232, no. 2, pp. 351-360, 2009.

[21] M. van Berkel et al., "Heat flux reconstruction and effective diffusion estimation from perturbative experiments using advanced filtering and confidence analysis," Nucl. Fusion, vol. 58, no. 9, p. 096036, 2018.

[22] A. Quarteroni and A. Valli, Numerical Approximation of Partial Differential Equations. Berlin; Heidelberg, Germany: Springer, 2008.

[23] I. Markovsky and S. Van Huffel, "Overview of total least-squares methods," Signal Process., vol. 87, no. 10, pp. 2283-2302, 2007.

[24] R. J. R. van Kampen, A. Das, S. Weiland, and M. van Berkel, "Complex Gaussian Process Regression for estimating spatially varying coefficients in heat transport," in 39th Benelux Meet. Syst. Control, p. 38,2020 .

[25] A. Ho et al., "Application of Gaussian process regression to plasma turbulent transport model validation via integrated modelling," Nucl. Fusion, vol. 59, no. 5, p. 056007, 2019.

[26] M. van Berkel, G. Vandersteen, H. Zwart, D. Hogeweij, and M. De Baar, "Maximum Likelihood Estimation of diffusion and convection in tokamaks using infinite domains," Proc. IEEE Int. Conf. Control Appl., pp. 1230-1234, 2013.

[27] C. de Boor, A Practical Guide to Splines. New York, USA: Springer 1978. 